\title{
Boundedness of Faber operators
}

\section{Yunus Emre Yıldırır and Ramazan Çetintaş}

"Correspondence:
yildirir@balikesir.edu.tr
Department of Mathematics,
Necatibey Faculty of Education,
Balikesir University, Balikesir, 10100,
Turkey

${ }^{*}$ Correspondence: yildirir@balikesir.edu.tr Necatibey Faculty of Education, Turkey

\begin{abstract}
In this work, we prove the boundedness of the Faber operators that transform the Hardy-Orlicz class $H_{M}(\mathbf{D})$ into the Smirnov-Orlicz class $E_{M}(G)$.
\end{abstract}

MSC: $41 \mathrm{~A} 10 ; 42 \mathrm{~A} 10$

Keywords: Faber operator; Hardy-Orlicz class; Smirnov-Orlicz class

\section{Introduction and main results}

Let a bounded simply connected domain $G$ with the boundary $\Gamma$ in the complex plane be given, such that the complement of the closed domain $G \cup \Gamma$ is a simply connected domain $G^{-}$, i.e., $G:=\operatorname{int} \Gamma$ and $G^{-}=\operatorname{ext} \Gamma$. Without loss of generality, we may assume $0 \in G$. Let $\mathbf{T}=\{w \in \mathbb{C}:|w|=1\}, \mathbf{D}=\operatorname{int} \mathbf{T}$ and $\mathbf{D}^{-}=\operatorname{ext} \mathbf{T}$.

By the Riemann theorem on a conformal mapping, there exists a unique function $w=$ $\Phi(z)$ meromorphic in $G^{-}$which maps the domain $G^{-}$conformally and univalently onto the domain $\mathbf{D}^{-}$and satisfies the conditions

$$
\Phi(\infty)=\infty \text { and } \lim _{z \rightarrow \infty} \frac{\Phi(z)}{z}=\gamma>0 .
$$

Let the function $z=\Psi(w)$ be the inverse function for $w=\Phi(z)$. This function maps the domain $\mathbf{D}^{-}$conformally and univalently onto the domain $G^{-}$.

Condition (1) implies that the function $w=\Phi(z)$, being analytic in the domain $G^{-}$without the point $z=\infty$, has a simple pole at the point $z=\infty$. Therefore its Laurent expansion in some neighborhood of the point $\infty$ has the form

$$
\Phi(z)=\gamma z+\gamma_{0}+\frac{\gamma_{1}}{z}+\cdots+\frac{\gamma_{k}}{z^{k}}+\cdots
$$

For a non-negative integer $n$, we can write

$$
\Phi^{n}(z)=F_{n}(z)+E_{n}(z), \quad z \in G^{-},
$$

where $F_{n}(z)$ is a polynomial of order $n$ and $E_{n}(z)$ is the sum of the infinite number of terms with negative powers.

The polynomial $F_{n}(z)$ is called the Faber polynomial of order $n$ for the domain $G$.

Let $h$ be a continuous function on $[0,2 \pi]$. Its modulus of continuity is defined by

$$
w(t, h):=\sup \left\{\left|h\left(t_{1}\right)-h\left(t_{2}\right)\right|: t_{1}, t_{2} \in[0,2 \pi],\left|t_{1}-t_{2}\right| \leq t\right\}, \quad t \geq 0 .
$$

(c) 2013 Yıldırır and Çetintaş; licensee Springer. This is an Open Access article distributed under the terms of the Creative Commons Attribution License (http://creativecommons.org/licenses/by/2.0), which permits unrestricted use, distribution, and reproduction in any medium, provided the original work is properly cited. 
The function $h$ is called Dini-continuous if

$$
\int_{0}^{\pi} t^{-1} w(t, h) d t<\infty
$$

The curve $\Gamma$ is called Dini-smooth if it has a parametrization

$$
\Gamma: \varphi_{0}(\tau), \quad 0 \leq \tau \leq 2 \pi
$$

such that $\varphi_{0}^{\prime}(\tau)$ is Dini-continuous and $\neq 0[1]$.

If $\Gamma$ is Dini-smooth, then

$$
0<c_{1} \leq\left|\Phi^{\prime}(z)\right| \leq c_{2}<\infty, \quad z \in \Gamma
$$

for some constants $c_{1}$ and $c_{2}$ independent of $z$.

A continuous and convex function $M:[0, \infty) \rightarrow[0, \infty)$ which satisfies the conditions $M(0)=0, M(x)>0$ for $x>0$,

$$
\lim _{x \rightarrow 0} \frac{M(x)}{x}=0, \quad \lim _{x \rightarrow \infty} \frac{M(x)}{x}=\infty
$$

is called an $N$-function.

The complementary $N$-function to $M$ is defined by

$$
N(y):=\max _{x \geq 0}(x y-M(x)), \quad y \geq 0 .
$$

Let $M$ be an $N$-function and $N$ be its complementary function. By $L_{M}(\Gamma)$ we denote the linear space of Lebesgue measurable functions $f: \Gamma \rightarrow \mathbb{C}$ satisfying the condition, for some $\alpha>0$,

$$
\int_{\Gamma} M[\alpha|f(z)|]|d z|<\infty
$$

The space $L_{M}(\Gamma)$ becomes a Banach space with the norm

$$
\|f\|_{L_{M}(\Gamma)}:=\sup \left\{\int_{\Gamma}|f(z) g(z)||d z|: g \in L_{N}(\Gamma), \rho(g ; N) \leq 1\right\},
$$

where $\rho(g ; N):=\int_{\Gamma} N[|g(z)|]|d z|$.

The norm $\|\cdot\|_{L_{M}(\Gamma)}$ is called Orlicz norm and the Banach space $L_{M}(\Gamma)$ is called Orlicz space. Every function in $L_{M}(\Gamma)$ is integrable on $\Gamma$ (see [2, p.50]), i.e., $L_{M}(\Gamma) \subset L_{1}(\Gamma)$.

Let $\mathbf{D}$ be a unit disk and $\Gamma_{r}$ be the image of the circle $\{w \in \mathbb{C}:|w|=r, 0<r<1\}$ under some conformal mapping of $\mathbf{D}$ onto $G$, and let $M$ be an $N$-function.

The class of functions which are analytic in $G$ and satisfy the condition

$$
\int_{\Gamma_{r}} M[|f(z)|]|d z|<\infty
$$

uniformly in $r$ is called the Smirnov-Orlicz class and denoted by $E_{M}(G)$. 
The Smirnov-Orlicz class is a generalization of the familiar Smirnov class $E_{p}(G)$. In particular, if $M(x):=x^{p}, 1<p<\infty$, then Smirnov-Orlicz class $E_{M}(G)$ determined by $M$ coincides with the Smirnov class $E_{p}(G)$.

Since (see [3]) $E_{M}(G) \subset E_{1}(G)$, every function in the class $E_{M}(G)$ has the nontangential boundary values a.e. on $\Gamma$ and the boundary value function belongs to $L_{M}(\Gamma)$. Hence $E_{M}(G)$ norm can be defined as

$$
\|f\|_{E_{M}(G)}:=\|f\|_{L_{M}(\Gamma)}, \quad f \in E_{M}(G) .
$$

Let $M:[0, \infty) \rightarrow[0, \infty)$ be an $N$-function. The class of functions which are analytic in $D$ and satisfy the condition

$$
\int_{0}^{2 \pi} M\left[\left|f\left(r e^{i t}\right)\right|\right] d t<\infty
$$

uniformly in $r$ is called the Hardy-Orlicz class and denoted by $H_{M}(\mathbf{D})$.

Since $H_{M}(\mathbf{D}) \subset H_{1}(\mathbf{D})$, every function in the class $H_{M}(\mathbf{D})$ has the nontangential boundary values a.e. on $\mathbf{T}$ and the boundary value function belongs to $L_{M}(\mathbf{T})$. Hence $H_{M}(\mathbf{D})$ norm can be defined as

$$
\|f\|_{H_{M}(\mathbf{D})}:=\|f\|_{L_{M}(\mathbf{T})}, \quad f \in H_{M}(\mathbf{D}) .
$$

The spaces $H_{M}(\mathbf{D})$ and $E_{M}(G)$ are Banach spaces respectively with the norm $\|f\|_{L_{M}(\mathbf{T})}$ and $\|f\|_{L_{M}(\Gamma)}$.

Hölder's inequality

$$
\int_{\Gamma}|f(z) g(z)||d z| \leq\|f\|_{L_{M}(\Gamma)}\|g\|_{L_{N}(\Gamma)}
$$

holds for every $f \in L_{M}(\Gamma)$ and $g \in L_{N}(\Gamma)$ [4, p.80].

Let $\Gamma$ be a Dini-smooth curve, $G$ be a finite domain bounded by $\Gamma$ and $\varphi \in H_{M}(\mathbf{D})$.

The Cauchy-type integral

$$
\left(F_{0} \varphi\right)(z)=\frac{1}{2 \pi i} \int_{\Gamma} \frac{\varphi(\Phi(\zeta))}{\zeta-z} d \zeta, \quad z \in G
$$

is called Faber operator for the domain $G$ from $H_{M}(\mathbf{D})$ into $E_{M}(G)$.

The inverse Faber operator from $E_{M}(G)$ into $H_{M}(\mathbf{D})$ is defined as

$$
\left(F_{1} f\right)(w)=\frac{1}{2 \pi i} \int_{|t|=1} \frac{f[\Psi(t)]}{t-w} d t, \quad|w|<1 .
$$

Let $\Gamma$ be a Dini-smooth curve and $G$ be a finite domain bounded by $\Gamma$. Then the boundedness of the Faber operators from $H_{p}(\mathbf{D})$ into $E_{p}(G)(p \geq 1)$ was proved in [5, p.125].

In this paper, we obtain the following results about the boundedness of the Faber operator from $H_{M}(\mathbf{D})$ into $E_{M}(G)$ and about the boundedness of the inverse Faber operator from $E_{M}(G)$ into $H_{M}(\mathbf{D})$. 
Theorem 1 Let $G$ be a finite domain bounded by a Dini-smooth curve $\Gamma$. Then the Faber operator $F_{0}: H_{M}(\mathbf{D}) \rightarrow E_{M}(G)$ has a finite norm and

$$
\left\|\left(F_{0} \varphi\right)\right\|_{E_{M}(G)} \leq\left\|F_{0}\right\|\|\varphi\|_{H_{M}(\mathbf{D})} .
$$

Theorem 2 Let $G$ be a finite domain bounded by a Dini-smooth curve $\Gamma$. Then the inverse Faber operator $F_{1}: E_{M}(G) \rightarrow H_{M}$ has a finite norm and

$$
\left\|\left(F_{1} f\right)\right\|_{H_{M}(\mathbf{D})} \leq\left\|F_{1}\right\|\|f\|_{E_{M}(G)} .
$$

Corollary 1 Let $G$ be a finite domain bounded by a Dini-smooth curve $\Gamma$ and $P_{n}$ be the image of the polynomial $\varphi_{n}$ defined in the unit disk under the Faber operator. Then

$$
\left\|\left(F_{0} \varphi\right)-P_{n}\right\|_{E_{M}(G)} \leq\left\|F_{0}\right\|\left\|\varphi-\varphi_{n}\right\|_{H_{M}(\mathbf{D})} .
$$

Corollary 2 Let $G$ be a finite domain bounded by a Dini-smooth curve $\Gamma$ and $\varphi_{n}$ be the image of the polynomial $P_{n}$ defined in $G$ under the inverse Faber operator. Then

$$
\left\|\left(F_{1} f\right)-\varphi_{n}\right\|_{H_{M}(\mathbf{D})} \leq\left\|F_{1}\right\|\left\|f-P_{n}\right\|_{E_{M}(G)} .
$$

With the help of these two corollaries, one can carry over the direct and inverse theorems on the order of the best approximations in mean, from the unit disk to the case of a domain with a sufficiently smooth boundary.

\section{Proof of the main results}

Proof of Theorem 1 For the Faber operator $\left(F_{0} \varphi\right)(z)$, the equality

$$
\left(F_{0} \varphi\right)(z)=\varphi(\Phi(z))+\frac{1}{2 \pi i} \int_{|t|=1} \varphi(t) F(t, \Phi(z)) d t
$$

holds [5, p.123], where

$$
F(t, w)=\frac{\Psi^{\prime}(t)}{\Psi(t)-\Psi(w)}-\frac{1}{t-w}, \quad|t| \geq 1,|w| \geq 1 .
$$

From (2) we obtain

$$
\begin{aligned}
& \int_{\Gamma}\left|\left(F_{0} \varphi\right)(z) g(z)\right||d z| \\
& \quad \leq \int_{\Gamma}|\varphi(\Phi(z)) g(z)||d z|+\frac{1}{2 \pi i} \int_{\Gamma}\left[\int_{|t|=1}|\varphi(t)||g(z)||F(t, \Phi(z))||d t|\right]|d z| .
\end{aligned}
$$

Using the definition of the Orlicz norm, Hölder's inequality and (3), we get

$$
\left\|\left(F_{0} \varphi\right)\right\|_{E_{M}(G)} \leq\left(m_{0}(\Gamma)+m_{1}(\Gamma)\right)\|\varphi\|_{H_{M}(\mathbf{D})}
$$

where

$$
m_{0}(\Gamma)=\sup _{|w|=1}\left|\Psi^{\prime}(w)\right|, \quad m_{1}(\Gamma)=\frac{1}{2 \pi i} \int_{\Gamma}|g(z)|\|F\|_{H_{N}(\mathbf{D})}|d z| .
$$


Therefore we obtain that

$$
\begin{aligned}
& \left\|\left(F_{0} \varphi\right)\right\| \leq\left(m_{0}(\Gamma)+m_{1}(\Gamma)\right) \text { and } \\
& \left\|\left(F_{0} \varphi\right)\right\|_{E_{M}(G)} \leq\left\|F_{0}\right\|\|\varphi\|_{H_{M}(\mathbf{D}) .}
\end{aligned}
$$

Proof of Theorem 2 For the inverse Faber operator $\left(F_{1} f\right)(w)$, the equality

$$
\left(F_{1} f\right)(w)=f(\Psi(z))-\frac{1}{2 \pi i} \int_{|t|=1} f(\Psi(t)) F(t, w) d t
$$

holds [5, p.127]. With the help of this equality, Theorem 2 is proved by the similar method of the proof of Theorem 1 .

\section{Competing interests}

The authors declare that they have no competing interests.

\section{Authors' contributions}

The author YEY determined the problem after making the literature research and organized the proofs of the theorems.

The author RÇ helped to the proofs of the theorems and wrote the manuscript in the latex.

Received: 14 December 2012 Accepted: 4 May 2013 Published: 21 May 2013

\section{References}

1. Israfilov, DM, Oktay, B, Akgün, R: Approximation in Smirnov-Orlicz classes. Glas. Mat. 40(60), 87-102 (2005)

2. Rao, MM, Ren, ZD: Theory of Orlicz Spaces. Dekker, New York (1991)

3. Kokilashvili, V: On analytic functions of Smirnov-Orlicz classes. Stud. Math. 31, 43-59 (1968)

4. Krasnoselskii, MA, Rutickii, YB: Convex Functions and Orlicz Spaces. Noordhoff, Groningen (1961)

5. Suetin, PK: Series of Faber Polynomials. Gordon \& Breach, New York (1988)

doi:10.1186/1029-242X-2013-257

Cite this article as: Yıldırır and Çetintaş: Boundedness of Faber operators. Journal of Inequalities and Applications 2013 2013:257.

\section{Submit your manuscript to a SpringerOpen ${ }^{\ominus}$ journal and benefit from:}

- Convenient online submission

- Rigorous peer review

Immediate publication on acceptance

- Open access: articles freely available online

- High visibility within the field

- Retaining the copyright to your article 